\title{
Manchons-trayeurs pour vaches : effets du diamètre et de l'élasticité du corps sur les caractéristiques de traite et sur quelques mesures de l'extrémité du trayon
}

\author{
J. Le Du ${ }^{1}$ et M. Taverna ${ }^{2}$ \\ avec la collaboration technique de F.A. de la Chevalerie et Y. Dano \\ 1 INRA, laboratoire de rechercines sur la traite, 65, rue de Saint-Brieuc, 35042 Rennes, France; \\ 2 INTA, station d'expérimentation agronomique 2300, Rafaela, Argentine
}

(reçu le 5 janvier 1989, accepté le 23 mars 1989)

Résumé - Selon le plan en carré latin $6 \times 6,18$ vaches ont été traites pendant 6 périodes d'une semaine avec 6 manchons-trayeurs expérimentaux qui diffèrent entre eux seulement par le diamètre et l'élasticité du corps. Deux modèles de manchons sont utilisés : petit et grand diamètre intérieur. Chaque modèie est moulé en 3 versions différentes : souple, normale (commercialisée) et dure avec des parois de 1,3; 2,3 et 3,3 mm d'épaisseur.

La cinétique d'écoulement du lait pendant la traite est enregistrée automatiquement. Avant et après la traite, on mesure le diamètre du trayon avec un pied à coulisse et l'épaisseur de son extrémité avec un compas d'épaisseur à ressort qui comprime les tissus. Les temps de traite machine et total sont significativement $(P<0,001)$ réduits (jusqu'à $16,3 \%$ ), par l'utilisation de manchons de grand diamètre et de dureté importante, ce qui n'est pas sans conséquences pratiques pour l'éleveur.

Dans nos conditions expérimentales, le diamètre du corps du manchon a plus d'influence que son élasticité. Compte tenu de leurs caractéristiques, il est probable que cette conclusion est également valable pour les manchons actuellement commercialisés. Les volumes de lait machine et total ne sont pas significativement affectés par les traitements. Après la traite, le diamètre du trayon et l'épaisseur des tissus de son extrémité sont plus faibles qu'avant la traite. Ces mesures semblent dépendre beaucoup des caractéristiques du matériel utilisé. Ainsi, après la traite, le diamètre du trayon est plus élevé lorsque la traite est effectuée avec les manchons de grand diamètre $(P<0,01)$. En outre, il existe des modifications significatives de l'épaisseur de l'extrémité du trayon $(P<0,01)$ qui tend à augmenter lorsque le diamètre du corps du manchon et sa dureté sont accrus. Ce travail mérite d'être poursuivi pour expliquer ces effets qui semblent associés à une traite rapide et pourraient être dus à l'accumulation de fluides dans l'extrémité du trayon.

vache - traite - trayon - manchon-trayeur

\footnotetext{
Summary - Teatcup liners for cows : effects of barrel diameter and stiffness on milking characteristics and teat-end condition after milking. The milking characteristics and teat-end condition after milking were compared for 2 types of teatcup liners differing in inner barrel diameter : narrow and wide bore. For each type of liner, 3 models differing in wall thickness $(1.3 ; 2.3 ; 3.3 \mathrm{~mm})$ and thus in barrel stiffness, were used : soft, normal (commercialized) and hard. A $6 \times 6$ latin square with 6 groups of 3 cows was used.

For the milking characteristics, the significant differences concerned mainly total and machine time which decreased with wide bore liners and with increasing stiffness of the barrel quantified by the measurement of its buckling pressure. The overall decrease which reached $16.3 \%$ for the total
} 
time may have practical consequences for the farmer. Under our experimental conditions, the bore of the barrel has a greater influence than its stiffness. Considering their characteristics, it seems that this conclusion applies also to the liners which are on the market.

The machine and total milk yield remained unaffected by the treatments. The diameter of the teats and the thickness of the teat end (measured under compression with spring-loaded calipers) were lower after milking than before milking. It seems that these measurements are highly dependent on the milking equipment. Thus, after milking, the teat diameter was higher with the wide bore liners $(P<0.01)$. Besides, there were significant variations in the teat end thickness $(P<0.01)$ which tended to increase with increasing stiffness and bore of the liner. Further studies are needed to explain these effects which seem associated with fast milking conditions and might be due to an accumulation of fluid in the teat-end.

cow - milking - teat - teacup liner

\section{INTRODUCTION}

La conception du manchon (dimensions, forme et élasticité) a un effet marqué sur les conditions d'écoulement du lait pendant la traite : débits et temps de traite, volume du lait obtenu par la machine seule, volume du lait obtenu au cours de l'cégouttage» (Le Du et Richard, 1977; O'Shea et O'Callaghan, 1980; Mayntz et Laidig, 1983; Le Du et Billon, 1986). Etant le seul composant de la machine à traire en contact avec l'animal, il pourrait également exercer une action sur les tissus du trayon dont la température, la couleur et l'élasticité sont plus ou moins modifiées après la traite (Schneider, 1980; Hamann, 1985; Isaksson, 1986; Hamann \& Mein, 1988).

Une approche théorique du fonctionnement du manchon étayée par de nombreux arguments expérimentaux montre que tous ces effets dépendraient principalement des pressions qu'il applique au trayon pendant la traite et des déformations subies par les tissus (Williams \& Mein, 1982; Mein \& Williams, 1984).

Ainsi, avec les modèles commercialisés, des études comparatives montrent que le débit d'écoulement du lait augmente lorsque le corps est dur et de grand diamètre (Le Du \& Richard, 1977; O'Shea \& O'Callaghan, 1980). Toutefois, les modèles de ce type auraient tendance à accroître la fréquence des lésions du trayon (Hamann, 1987) et des infections mammaires (O'Shea, 1987).

Toutefois, ces travaux ne dissocient pas les effets respectifs des caractéristiques dimensionnelles du manchon par exemple, le diamètre du corps - et des propriétés fonctionnelles - par exemple, l'élasticité du corps - (McGrath \& O'Shea, 1972, chez la vache; Le Du et al., 1978; Le Du, 1982, chez la brebis).

Pour approfondir cette question, notre étude a donc pour objet de comparer entre eux 6 manchons-trayeurs expérimentaux qui diffèrent entre eux seulement par le diamètre ou l'élasticité du corps.

\section{MATÉRIEL ET MÉTHODES}

\section{Equipement et technique de traite}

Deux modèles de manchons de marque AlfaLaval (Tumba, Suède) montés dans des gobelets $n^{\circ} 960$ 550-80 sont utilisés (Tableau I) : $\mathrm{n}^{\circ}$ 960018-01 de petit diamètre intérieur (22,3 $\mathrm{mm}$, code $P$ ) et $n^{\circ}$ 960016-01 de grand diamètre $(24,6 \mathrm{~mm}$, code $\mathrm{G})$. Chaque modèle de manchon est moulé en 3 versions différentes : 
souple, normale (version commerciale) et dure. Elles diffèrent entre elles seulement par l'épaisseur des parois $(1,3 ; 2,3$ et $3,3 \mathrm{~mm})$ et donc par l'élasticité du corps qui est appréciée en mesurant la pression de flambage du manchon selon la technique décrite par Le Du et al. (1984).

La traite est effectuée à $8 \mathrm{~h}$ et $16 \mathrm{~h}$ dans une installation de type tandem équipée de bocaux mesureurs avec un lactoduc situé sous le quai. Les principales caractéristiques du faisceau-trayeur sont les suivantes : pulsation simultanée de fréquence $60 \mathrm{p} . / \mathrm{min}$ avec un rapport de pulsation de $51 \%$ et un niveau de vide nominal de $46 \mathrm{kPa}$; griffe $\mathrm{n}^{\circ} 21142$ de marque Fullwood (Ellesmere, Grande-Bretagne) d'un volume de $126 \mathrm{ml}$ avec une admission d'air de $7 \mathrm{l} / \mathrm{min}$, faisceau-trayeur d'un poids de $2,6 \mathrm{~kg}$ (sans les tuyaux à lait et de pulsation reliant la griffe au bocal mesureur).

Pour contrôler l'état sanitaire des animaux, chaque semaine le mercredi et le jeudi, des numérations cellulaires sont effectuées au compteur Coulter sur le volume de lait total de la traite du soir.

\section{Protocole expérimental}

Suivant un carré latin $6 \times 6,18$ vaches de race française Frisonne Pie Noire sont réparties en 6 lots. Au début de l'expérimentation, les stades de lactation sont de 20 à 206 jours (moyenne de $60 \mathrm{j}$ ) et les productions laitières de 16 à 34,5 I par jour (moyenne de 26,1 I). Chaque lot est trait durant 6 périodes de 7 jours avec chacun des 6 manchons expérimentaux. Pour chaque traitement, les mesures suivantes sont effectuées sur chaque vache :

- enregistrement des caractéristiques des traites : à la traite du matin, le $5^{\mathrm{e}}, 6^{\mathrm{e}}$ ou $7^{\mathrm{e}}$ jour de chaque période,

- mesures sur l'extrémité du trayon : à la traite du soir le $5^{\mathrm{e}}$ ou $6^{\mathrm{e}} \mathrm{j}$ de chaque période.

Les effets directs et résiduels des traitements sont estimés en répétant durant une septième période d'une semaine les traitements de la sixième semaine (Cochran et Cox, 1962). En conséquence, nous présentons des résultats ajustés de façon à éliminer les effets résiduels des traitements précédents.

Tableau I. Principales caractéristiques physiques des 6 manchons-trayeurs. (1) Pour chacun des 2 types de manchons, les lettres $s$, $n$ et $d$ repèrent les versions souples, normales et dures. (2) Pression de flambage : vide à appliquer à l'intérieur du manchon monté dans son gobelet pour que 2 faces opposées entrent en contact sous le trayon lors de l'aplatissement. Elle est mesurée après 24 heures sous pulsation dans des conditions standardisées décrites par Le Du et al. (1984). (3) Mesuré à $75 \mathrm{~mm}$ de l'embouchure.

Manchons

(1)

\begin{tabular}{lllllll}
\hline & Ps & 2,4 & 5,6 & 22,3 & 1,3 & 19,6 \\
$\begin{array}{l}\text { Petit diamètre } \\
\text { (Code P) }\end{array}$ & Pn & 4,5 & 13,7 & 22,3 & 2,3 & 19,6 \\
& Pd & 6,0 & 27,6 & 22,3 & 3,3 & 19,6 \\
& Gs & 2,5 & 5,0 & 24,6 & 1,3 & 23,6 \\
$\begin{array}{l}\text { Grand diamètre } \\
\text { (Code G) }\end{array}$ & Gn & 4,8 & 12,3 & 24,6 & 2,3 & 23,6 \\
& Gd & 6,5 & 24,9 & 24,6 & 3,3 & 23,6
\end{tabular}

\section{Tension Pression Diam. Int. Epaisseur Diamètre}

(kg) flambage ducorps parois embouchure

$\begin{array}{lllll} & (\mathrm{kPa}) & (\mathrm{mm}) & (\mathrm{mm}) & (\mathrm{mm}) \\ (2) & (2) & (3) & (3) & \text { (3) }\end{array}$




\section{Enregistrement des caractéristiques de traite}

Les volumes de lait recueillis durant la traite sont lus toutes les $20 \mathrm{~s}$ à l'aide d'un équipement automatique d'enregistrement décrit par Le Du (1984).

Les critères mesurés à partir de ces contrôles sont les suivants : volume de lait machine (jusqu'à la période de $20 \mathrm{~s}$ où le débit devient inférieur à $0,2 \mathrm{l} / \mathrm{min}$ ), volume du lait d'égouttage et du lait total (en l); temps de traite machine et total (en min); débit du lait machine et débit total (en $\mathrm{l} / \mathrm{min}$ ); débit maximum défini comme étant le volume maximum de lait recueilli durant 2 intervalles consécutifs de $20 \mathrm{~s}$ (en $1 / \mathrm{min}$ ).

\section{iwesures sur l'extrémité du trayon}

Les mesures sont effectuées avant la préparation du pis pour la traite et aussitôt après la traite sur les deux trayons avant :

- le diamètre nominal $(\mathrm{mm})$ est mesuré à 30 $\mathrm{mm}$ de l'extrémité du trayon avec un pied à coulisse;

- l'épaisseur des tissus (duromètre) de l'extrémité du trayon $(\mathrm{mm})$ est mesurée à l'aide d'un compas d'épaisseur à ressort selon la technique décrité par Le Du \& Benmederbel (1984). L'extrémité du trayon est introduite entre deux disques de diamètre $30 \mathrm{~mm}$ qui appliquent au trayon une force de compression de 2,35 kg environ. La rigidité est définie comme étant l'épaisseur des tissus comprimés;

Tableau II. Classement des manchons en fonction des caractéristiques de traite. Les différences sont significatives au seuil de ${ }^{* \star *}: 0,001 ;{ }^{* *}: 0,01 ;{ }^{*}: 0,05$ et $\mathrm{ns}:$ non significatives au seuil $P=0,05$. Les valeurs soulignées d'un même trait ne diffèrent pas entre elles au seuil 0,05 (test de Duncan).

\begin{tabular}{|c|c|c|c|c|c|c|c|}
\hline \multirow[b]{2}{*}{ Lait machine (1) } & \multicolumn{6}{|c|}{ Classement des manchons } & \multirow{2}{*}{$\frac{F}{1,45 \mathrm{~ns}}$} \\
\hline & $\begin{array}{l}\text { Gn } \\
15,66\end{array}$ & $\begin{array}{l}P n \\
16,40\end{array}$ & $\begin{array}{l}\mathrm{Gd} \\
16,46\end{array}$ & $\begin{array}{l}\text { Ps } \\
16,54\end{array}$ & $\begin{array}{l}\text { Gs } \\
16,68\end{array}$ & $\begin{array}{l}P d \\
16,70\end{array}$ & \\
\hline Lait d'égouttage (l) & $\begin{array}{l}\text { Gs } \\
0,40\end{array}$ & $\begin{array}{l}\text { PS } \\
0,44\end{array}$ & $\begin{array}{l}\mathrm{Pd} \\
0,50\end{array}$ & $\underset{0,55}{\mathrm{Gd}}$ & $\begin{array}{l}P n \\
0,56\end{array}$ & $\underset{0,59}{G n}$ & $0,89 \mathrm{~ns}$ \\
\hline Lait total (l) & $\begin{array}{l}\text { Gn } \\
16,20\end{array}$ & $\begin{array}{l}\text { Gd } \\
16,98\end{array}$ & $\begin{array}{l}\mathrm{Pn} \\
17,02\end{array}$ & $\begin{array}{l}\text { Ps } \\
17,03\end{array}$ & $\begin{array}{l}\text { Gs } \\
17,08\end{array}$ & $\begin{array}{l}\mathrm{Pd} \\
17,20\end{array}$ & $1,25 \mathrm{~ns}$ \\
\hline Temps machine (min) & $\begin{array}{l}\mathrm{Gd} \\
6,31\end{array}$ & $\underset{6,51}{G n}$ & $\begin{array}{l}\text { Gs } \\
6,84\end{array}$ & $\begin{array}{l}\mathrm{Pd} \\
7,20\end{array}$ & $\begin{array}{l}\mathrm{Pn} \\
7,27\end{array}$ & $\begin{array}{l}\text { Ps } \\
7,74\end{array}$ & $5,05^{* * *}$ \\
\hline Temps total (min) & $\begin{array}{l}\mathrm{Gd} \\
7,25\end{array}$ & $\underset{7,51}{G n}$ & $\begin{array}{l}\text { Gs } \\
7,62\end{array}$ & $\begin{array}{l}\mathrm{Pd} \\
8,10\end{array}$ & $\begin{array}{l}\mathrm{Pn} \\
8,32\end{array}$ & $\begin{array}{l}\text { Ps } \\
8,66\end{array}$ & $5,13^{* * *}$ \\
\hline Débit machine $1 / \min$ ) & $\begin{array}{l}\text { Ps } \\
2,23\end{array}$ & $\begin{array}{l}\mathrm{Pn} \\
2,33\end{array}$ & $\underset{2,47}{\mathrm{Gn}}$ & $\begin{array}{l}\text { Gs } \\
2,47\end{array}$ & $\begin{array}{l}\mathrm{Pd} \\
2,51\end{array}$ & $\begin{array}{c}\mathrm{Gd} \\
2,69\end{array}$ & $4,90^{* * *}$ \\
\hline Débit total ( $1 / \mathrm{min})$ & $\begin{array}{l}\text { Ps } \\
2,02\end{array}$ & $\begin{array}{l}\mathrm{Pn} \\
2,05\end{array}$ & $\begin{array}{l}\mathrm{Gn}_{2,21} \\
\end{array}$ & $\begin{array}{l}\mathrm{Pd} \\
2,23\end{array}$ & $\begin{array}{l}\text { Gs } \\
2,25\end{array}$ & $\begin{array}{l}\mathrm{Gd} \\
2,40\end{array}$ & $4,97^{\star \star *}$ \\
\hline Débit maximum $(1 / \mathrm{min})$ & $\begin{array}{l}\text { Ps } \\
3,70\end{array}$ & $\begin{array}{l}\mathrm{Pn} \\
3,89\end{array}$ & $\mathrm{Gn}_{3,91}$ & $\begin{array}{l}\text { Gs } \\
4,00\end{array}$ & $\begin{array}{l}\mathrm{Gd} \\
4,10\end{array}$ & $\begin{array}{l}\mathrm{Pd} \\
4,18\end{array}$ & $2,67^{*}$ \\
\hline
\end{tabular}


- l'aplatissement du trayon ( $\mathrm{mm}$ ) est égal à la différence entre le diamètre nominal et l'épaisseur des tissus comprimés.

\section{RÉSULTATS ET DISCUSSION}

\section{Effets sur les caractéristiques de traite}

Dans nos conditions expérimentales, le manchon n'affecte pas de façon significative les productions totales de lait (Tableau II). Ceci confirme tous nos résultats antérieurs notamment ceux de Le Du \& Richard (1977) pourtant obtenus en comparant des modèles commerciaux très différents. Par contre, ces auteurs montraient que le manchon modifiait les proportions du lait obtenues respectivement par la machine seule et lors de l'opération d'égouttage : une augmentation du volume de lait machine entraînait une diminution du lait d'égouttage qui représentait $22 \%$ du lait total avec le plus mauvais manchon contre $6 \%$ avec le meilleur modèle. Nous n'observons pas cet effet. D'une part, les 6 manchons sont de conception plus homogène qu'en 1977, au moins dans leurs caractéristiques dimensionnelles. Par ailleurs, le choix des paramètres de traite - diminution du rapport de pulsation de 75 à $51 \%$ et du niveau de vide de 50 à $46 \mathrm{kPa}$ concourt à abaisser le volume d'égouttage. II en résulte que celui-ci très faible quel que soit le manchon, représente seulement 2,4 à $3,8 \%$ du volume de lait total et ne varie pas de façon significative.

Le classement des manchons montre que leurs caractéristiques influencent principalement les temps de traite $(P<0,001)$ qui sont plus élevés :
- avec les manchons de faible diamètre : en moyenne $13,5 \%$ pour le temps machine et $12,1 \%$ pour le temps total de plus qu'avec les modèles de grand diamètre,

- lorsqu'on diminue la pression de flambage, puisque l'accroissement moyen du temps machine iorsqu'on passe de la version dure à la version souple est de $7,5 \%$ pour $P$ contre $8,1 \%$ pour $G$. Pour le temps total, les chiffres sont respectivement 5,2 et $6,9 \%$.

Les volumes de lait obtenus avec les 6 manchons étant similaires, la variabilité des temps de traite correspondants conduit à observer des différences hautement significatives sur les débits machine et total respectivement accrus de 20,6 à $18,8 \%$ en passant du manchon de faible diamètre et souple (Ps) au manchon de grand diamètre et dur (Gd). Entre ces deux extrêmes, le classement des manchons est moins net qu'en ce qui concerne les temps de traite:

- en moyenne, les débits augmentent avec les manchons de grand diamètre : $+7,9 \%$ en moyenne pour le débit machine : $+8,9 \%$ pour le débit total,

- l'effet de la pression de flambage est moins marqué bien que les débits aient tendance à diminuer lorsqu'on diminue la pression de flambage.

Le débit maximum a tendance à varier de la même façon que les débits machine et total avec les caractéristiques des manchons. Toutefois, l'ampleur des variations est plus faible $(+13,0 \%)$ et les effets respectifs du diamètre du manchon et de la souplesse du corps sont peu apparents.

Ces résultats appellent plusieurs commentaires : Selon Thiel (1974), le corps du manchon influencerait les temps de traite et les débits d'écoulement du lait tandis que le degré de vidange du pis, et donc le volume d'égouttage, dépendraient de la conception de l'embouchure. Plus 
précisément, McGrath \& O'Shea (1972) sur des vaches puis Le Du et al. (1978) sur des brebis, ont démontré que le volume d'égouttage décroît d'autant plus que le diamètre de l'embouchure du manchon est grand par rapport à celui du corps. Or, pour nos deux modèles de manchons, l'écart entre ces deux dimensions est dans le même sens : le diamètre de l'embouchure plus faible que celui du corps doit être considéré comme étant du même ordre de grandeur : 2,7 mm pour $P$ contre $1,0 \mathrm{~mm}$ pour $G$, soit une différence de $1,7 \mathrm{~mm}$ seulement.

Les volumes d'égouttage, qui représentent au maximum 0,59 । ou $3,8 \%$ du lait sont probablement trop faibles pour que la vidange du pis soit significativement affectée. Ceci explique que les différences significatives entre les manchons concernent seulement les temps et les débits qui dépendent directement des caractéristiques du corps des manchonstrayeurs.
L'appréciation des effets respectifs du diamètre du corps du manchon et de sa pression de flambage est relativement délicate. En effet, en considérant les données brutes (Tableau II), il apparaît que la variation des temps de traite et des débits est de l'ordre de $20 \%$ en comparant les deux manchons extrêmes Gd et Ps (par exemple $+22,7 \%$ pour le temps machine avec Ps par rapport à $\mathrm{Gd}$ ). En moyenne, l'accroissement est de $+13,5 \%$ lorsqu'on compare un modèle $\mathrm{G}$ à un modèle P. II est de $+7,8 \%$ lorsqu'on compare une version dure à une version souple.

L'effet des caractéristiques dimensionnelles serait donc à priori près de 2 fois plus important que celui de la pression de flambage. En fait, si l'on considère les diamètres intérieurs - 22,3 et $24,6 \mathrm{~mm}-$, nos manchons sont de dimensions voisines et très proches du diamètre moyen que nous avions mesuré sur 36 manchons choisis parmi les modèles couramment commercialisés en France : dia-

Tableau III. Classement des manchons en fonction des mesures des trayons ( $\mathrm{mm}$ ). Les différences sont significatives au seuil de ${ }^{* * *}: 0,001 ;{ }^{* *}: 0,01$ et ns : non significatives au seuil 0,05 . Les valeurs soulignées d'un mème trait ne diffèrent pas entre elles au seuil 0,05 (test de Duncan).

\begin{tabular}{|c|c|c|c|c|c|c|c|c|}
\hline \multicolumn{2}{|c|}{ Mesures des trayons } & \multicolumn{6}{|c|}{ Classement des manchons } & \multirow{2}{*}{$\begin{array}{l}F \\
1,17 \mathrm{~ns}\end{array}$} \\
\hline $\begin{array}{l}\text { Avant la } \\
\text { traite }\end{array}$ & Diam. nominal & $\begin{array}{l}P n \\
25,29\end{array}$ & $\begin{array}{l}\mathrm{Pd} \\
25,37\end{array}$ & $\begin{array}{l}\text { Gs } \\
25,56\end{array}$ & $\begin{array}{l}\text { Ps } \\
25,65\end{array}$ & $\begin{array}{l}\text { Gd } \\
25,78\end{array}$ & $\begin{array}{l}\text { Gn } \\
25,84\end{array}$ & \\
\hline & Epais. tissus & $\begin{array}{l}\text { Pn } \\
9,48 \\
\end{array}$ & $\begin{array}{l}\text { Gn } \\
9,53\end{array}$ & $\begin{array}{l}\text { Ps } \\
9,56 \\
\end{array}$ & $\begin{array}{l}\text { Gs } \\
9,70 \\
\end{array}$ & $\begin{array}{l}\text { Gd } \\
9,72 \\
\end{array}$ & $\begin{array}{l}\mathrm{Pd} \\
9,77 \\
\end{array}$ & 1,49 ns \\
\hline & Aplatissement & $\begin{array}{l}\mathrm{Pd} \\
15,59\end{array}$ & $\begin{array}{l}\mathrm{Pn} \\
15,80\end{array}$ & $\begin{array}{l}\text { Gs } \\
15,86\end{array}$ & $\begin{array}{l}\text { Ps } \\
16,08\end{array}$ & $\begin{array}{l}G d \\
16,05\end{array}$ & $\begin{array}{l}\text { Gn } \\
16,31\end{array}$ & $1,24 \mathrm{~ns}$ \\
\hline \multirow[t]{3}{*}{$\begin{array}{l}\text { Après la } \\
\text { traite }\end{array}$} & Diam. nominal & $\begin{array}{l}P n \\
23,86\end{array}$ & $\begin{array}{l}P d \\
24,16\end{array}$ & $\begin{array}{l}\text { Ps } \\
24,33\end{array}$ & $\begin{array}{l}\text { Gs } \\
24,96\end{array}$ & $\begin{array}{l}G n \\
25,18\end{array}$ & $\begin{array}{l}\mathrm{Gd} \\
25,28\end{array}$ & $11,18^{* * *}$ \\
\hline & Epais. tissus & $\begin{array}{l}\text { Ps } \\
8,86\end{array}$ & $\begin{array}{l}\text { Gs } \\
8,91\end{array}$ & $\begin{array}{l}\mathrm{Pn} \\
8,94\end{array}$ & $\begin{array}{l}\text { Gn } \\
9,20\end{array}$ & $\begin{array}{l}\mathrm{Pd} \\
9,20 \\
\end{array}$ & $\begin{array}{l}\mathrm{Gd} \\
9,44\end{array}$ & $3,85 * *$ \\
\hline & Aplatissement & $\begin{array}{l}P n \\
14,92\end{array}$ & $\begin{array}{l}\mathrm{Pd} \\
14,95\end{array}$ & $\begin{array}{l}\text { Ps } \\
15,47\end{array}$ & $\begin{array}{l}\text { Gd } \\
15,84\end{array}$ & $\begin{array}{l}G n \\
15,98\end{array}$ & $\begin{array}{l}\text { Gs } \\
16,05\end{array}$ & $8,08^{* * *}$ \\
\hline
\end{tabular}


mètre de $23,7 \mathrm{~mm}$ en moyenne variant entre 21 et $28 \mathrm{~mm}$ (Le Du, 1983; Le Du et al., 1984). Par contre, la variabilité des pressions de flambage de nos manchons expérimentaux, comprises entre 5,0 et $27,6 \mathrm{kPa}$ est nettement plus élevée que celle des modèles commerciaux : 10,0 $\mathrm{kPa}$ en moyenne avec $95 \%$ des valeurs entre 8,2 et $11,7 \mathrm{kPa}$.

Finalement, cette analyse confirme l'influence prépondérante des caractéristiques dimensionnelles. Ainsi, le temps de traite machine croît en moyenne de $13 \%$ lorsque le diamètre diminue seulement de $9,3 \%$. Par contre, l'accroissement reste inférieur à $8 \%$ en passant d'un modèle souple à un modèle dur, c'est-à-dire en accroissant la pression de flambage de près de $400 \%$.

\section{Effets sur les mesures du trayon}

Nous ne constatons aucune différence entre les mesures effectuées sur le trayon avant la traite (Tableau III). Ceci tendrait à démontrer que les traitements ne produisent aucun effet rémanent susceptible de provoquer une modification des propriétés des tissus du trayon en fonction du manchon utilisé (par exemple, augmentation progressive de l'épaisseur des tissus). Toutefois, ces conclusions auraient peutêtre été différentes dans d'autres conditions expérimentales notamment avec des périodes plus longues, pendant une lactation complète ou avec des animaux à d'autres stades ou rangs de lactation (en particulier des génisses en début de lactation).

La comparaison des mesures effectuées avant et après la traite montre que le diamètre nominal et l'épaisseur des tissus diminuent respectivement de 0,96 et $0,52 \mathrm{~mm}$ en moyenne après la traite. Les diminutions sont plus importantes avec les manchons $P$ qu'avec les modèles $G$ : 0,60 contre $0,47 \mathrm{~mm}$ pour le diamètre (ns); 0,59 contre $1,32 \mathrm{~mm}$ pour l'épaisseur des tissus $(P<0,01)$ (Tableau III).

En utilisant une méthodologie similaire Schneider (1980) avait également relevé avec un matériel de traite conventionnel (semblable au nôtre) des diminutions de l'épaisseur des tissus du même ordre de grandeur que nous : $0,30 \mathrm{~mm}$. Toutefois, en changeant de matériel de traite, les mesures variaient de façon hautement significative $(P<0,01)$. Ainsi, l'épaisseur diminue de $1,40 \mathrm{~mm}$ avec le matériel Happel qui accroît le massage en admettant de l'air comprimé dans la chambre de pulsation. A l'inverse, l'épaisseur après la traite augmente en moyenne de $2,40 \mathrm{~mm}$ avec la machine PKME à succion permanente qui ne masse pas le trayon. Hamann (1985) observe également un accroissement de l'épaisseur des tissus avec le matériel PKME (+ $23 \%$ ) et une diminution en admettant de l'air comprimé dans la chambre de pulsation (- $6 \%$ ). Par contre, avec le matériel conventionnel, Hamann observe - non pas une diminution - mais un accroissement moyen de $8 \%$ de l'épaisseur des tissus.

Dans les deux cas, la température superficielle est accrue après la traite : $2,6{ }^{\circ} \mathrm{C}$ pour Hamann (1985); $2,0^{\circ} \mathrm{C}$ pour Schneider (1980). Au vu de l'ensemble de ces résultats, nous pensons que l'effet d'une machine à traire sur le trayon est extrêmement variable et dépendant des caractéristiques du matériel de traite.

D'une part, il existerait des conditions de traite "traumatisantes" qui sont celles des machines à traire sans pulsation du type PKME. L'accroissement de la rigidité des tissus après la traite et la diminution de la température sont selon Schneider (1980) les indices d'une hyperémie de congestion due à un ralentissement de l'écoulement du sang veineux. Elle se tra- 
duit par une couleur rouge voire violacée des tissus après la traite et par une augmentation plus ou moins perceptible de la dureté du trayon et de son volume. Ceci correspond aux observations faites par nos trayeurs en utilisant les manchons les plus durs.

A l'inverse, on obtient des conditions de traite "douces" lorsque l'effet de "massage" est accentué en introduisant par exemple de l'air comprimé dans la chambre de pulsation (système Happel). Selon Schneider (1980), "le trayon après la traite est nettement plus souple et plus chaud; sa couleur est d'un rouge-rose". Ces effets seraient caractéristiques de I'hyperémie active qui provoque une dilatation de toutes les voies sanguines par afflux de sang artériel.

Ces observations sont en parfait accord avec les conclusions de Williams \& Mein (1982); Mein \& Williams (1984) qui, analysant le fonctionnement du manchon, attribuent ses effets sur les conditions d'écoulement du lait (variation des débits) et l'état des trayons (variation de la rigidité des tissus) principalement à des phénomènes de congestion vasculaire de l'extrémité. Selon ces auteurs, avec une machine à traire conventionnelle, l'activité des muscles lisses ou les déformations de l'épithélium n'auraient qu'une incidence très marginale.

Nos périodes expérimentales d'une semaine étaient évidemment trop courtes pour apprécier rigoureusement l'incidence des manchons sur l'état sanitaire du pis. Toutefois, il est à noter que les animaux supportaient parfaitement d'être traits avec des manchons très différents (notamment en ce qui concerne leur dureté) et ne manifestaient pas de comportements anormaux pendant la traite lors des changements hebdomadaires de traitements. Enfin, l'état sanitaire des animaux ne semble pas s'être dégradé durant l'expérimentation. En $1^{\text {re }}$ semaine, la moyenne des numérations cellulaires par vache était de 183000 cellules par $\mathrm{ml}$ (contre 173000 en $7^{e}$ semaine) avec $30,5 \%$ des comptages a plus de 300000 (contre $25,0 \%$ en $7^{e}$ semaine).

\section{CONCLUSIONS}

Notre travail montre que les caractéristiques du corps du manchon-trayeur ont une incidence sur les débits d'écoulement du lait qui augmentent lorsqu'on accroît la pression de flambage ou le diamètre. La diminution du temps total de traite qui en résulte peut avoir des incidences pratiques pour l'éleveur puisqu'elle atteint $16 \%$ soit 1,41 min lorsqu'on agit à la fois sur les deux paramètres. Dans nos conditions expérimentales, bien que les diamètres des manchons soient peu différents $(22,3$ et $24,6 \mathrm{~mm}$; Tableau l), il apparaît clairement que cette caractéristique dimensionnelle a plus d'influence que l'élasticité du corps appréciée par sa pression de flambage. Compte tenu de leurs caractéristiques étudiées par Le Du et al. (1984), il est probable que ces conclusions peuvent être généralisées à l'ensemble des modèles moulés en caoutchouc actuellement commercialisés en France.

En contrepartie, avec des manchons conduisant à un débit élevé d'écoulement du lait, on observe des conditions de traite que nous avons qualifiées de "traumatisantes». En effet, quelle que soit leur pression de flambage avec les modèles les plus grands de type $\mathrm{G}$ le trayon apparaît "tuméfié" puisque leur diamètre mesuré après la traite est plus élevé qu'en trayant avec les manchons étroits de type P. L'incidence de la pression de flambage 
est beaucoup moins marquée que celle du diamètre. Toutefois, le classement des manchons montre que les modèles durs conduiraient aux conditions de traite les plus "traumatisantes" puisque l'épaisseur des tissus mesurée après la traite croît avec la pression de flambage.

II existe des différences hautement significatives $(P<0,01)$ entre les modèles commerciaux Gn et Pn (Tableau II) dont les pressions de flambage sont pourtant similaires : 12,3 et $13,7 \mathrm{kPa}$ (Tableau I). On observe qu'avec le modèle $\mathrm{Gn}$, le temps total de traite est diminué tandis que le diamètre nominal est accru ce qui indiquerait une traite plus traumatisante. Une étude de Bakke \& Binde (1984) donne des indications sur les effets à long terme. Ces auteurs ont suivi 13 troupeaux dans lesquels chacun des manchons $\mathrm{Gn}$ et $\mathrm{Pn}$ était utilisé pour traire la moitié des animaux. Qualitativement, cette étude confirme nos résultats. Au bout de 6 mois, avec le manchon $\mathrm{Gn}$, les trayons sont devenus significativement plus gros et plus longs sans que la sévérité des lésions du trayon (éversion du canal en particulier) soit affectée. Globalement, l'état sanitaire du pis a tendance à être meilleur avec le manchon Pn. Toutefois, les effets sont significatifs seulement pour les vaches en $1^{\text {re }}$ lactation : amélioration des résultats du CMT, diminution du nombre de quartiers infectés et du taux des nouvelles infections. II semble donc judicieux de choisir le manchon-trayeur prioritairement pour qu'il soit adapté aux animaux en $1^{\text {re }}$ lactation dont les trayons sont moins volumineux que ceux des multipares (Hamann, 1987).

II peut paraître surprenant de constater qu'avec tous les manchons, le diamètre du trayon et l'épaisseur des tissus mesurés en comprimant son extrémité sont plus faibles après la traite. L'étude de la bibliographie (Schneider, 1980; Hamann, 1985; Hamann \& Mein, 1988) montre que l'on peut également observer des effets inverses, c'est-à-dire un accroissement des mesures lorsque les conditions de traite sont plus "traumatisantes» que les nôtres (par exemple en trayant sans pulsation). On considère que ces modifications de l'épaisseur des parois du trayon après la traite sont probablement dues à l'accumulation plus ou moins importante de fluides dans son extrémité et il semble souhaitable d'éviter cet effet qui s'apparente à une congestion et/ou à un œdème (Hamann \& Mein, 1988).

En comparant les mesures du trayon effectuées avant et après la traite, on dispose donc d'une méthode simple pour apprécier l'effet local de la machine à traire à ce niveau (effet des composants, du réglage, etc.) et éventuellement porter un jugement plus général sur les conditions dans lequelles se déroule la traite (effet du massage du pis, de l'égouttage, de la surtraite, etc.). En outre, l'étude des variations individuelles entre animaux à différents stades de leur lactation peut donner des indications sur leur aptitude à être traits.

\section{REMERCIEMENTS}

Nous exprimons notre reconnaissance à la Société Alfa-Laval qui a fourni à titre gracieux les manchons prototypes, Mr. P. Billon de I'ITEB et le personnel de l'étable du Rheu pour la collaboration apportée à la réalisation de ce travail.

\section{RÉFÉRENCES}

Bakke H. \& Binde M. (1984) Effect of teatcup liner design on teat characteristics and udder health, Nord. Vet. med. 117-123 
Cochran W.G. \& Cox G.M. (1962) In : Experimental Designs, (Shewhar W.A. and Wilks S.S. eds.) Wiley \& Sons, London, pp. 133-142

Hamann J. (1985) Measurement of machine milking induced teat tissue reactions, Milchwissenschaft 40, 16-18

Hamann J. (1987) Effects of machine milking on teat end condition - a literature review. In : Machine milking and Mastitis. Bull. I.D.F. $n^{\circ}$ 215, Ed F.I.L, Brussels, 35-55

Hamann J. \& Mein G.A. (1988) Responses of the bovine teat to machine milking : measurement of changes in thickness of the teat apex. J. Dairy Res. 55, 331-338

Isaksson A. (1986) Right and wrong in the udder sphere, Vlaams Diergeeskd. Tijdschr. $55,244-250$

Le Du J. (1982) Comparaison de matériels de traite pour brebis : manchons en silicone ou en caoutchouc, gobelets en inox ou en plastique. Ann. Zootech. 32, 43-52

Le Du J. (1983) Caractéristiques des manchons-trayeurs pour vaches. Comparaison de manchons d'origine et adaptables. Rapport INRA, Rennes

Le Du J. (1984) Equipement pour l'enregistrement automatique de la cinétique de descente de lait. Proc. $3^{\text {rd }}$ Intern. Symp. on Mecanical Milking of Small Ruminants, Sever-Cuesta (eds.) Valladolid (Spain), pp. 425-532

Le Du J. \& Richard J. (1977) Influence du manchon-trayeur sur les caractéristiques de traite des vaches de race française Frisonne Pie Noire. Ann. Zootech. 26, 503-512

Le Du J. \& Benmederbel B. (1984) Aptitude des chèvres de race Saanen à la traite mécanique. Relations avec les caractéristiques physiques du trayon. Ann. Zootech. 33, 375-384

Le Du J. \& Billon P. (1986) Machine à traire et mammites : incidence du faisceau-trayeur. Journée Nationale d'information, Paris, (Ed. Comité Français Interprof. Traite Mécanique)

Le Du J., Labussière J., Douaire M. \& Combaud J.F. (1978) Effet de la conception de l'em- bouchure du manchon-trayeur sur les caractéristiques de traite des brebis Préalpes du Sud. Ann. Zootech. 27, 571-581

Le Du J., Arranz Arriola J. \& Dano Y. (1984) Comparative studies on physical characteristics of original and imitation teatcup liners. Ir. $J$. Agric. Res. 23, 89-96

Mayntz M. \& Laidig F. (1983) Untersuchungen zum Einfluss des Zitzengummikopfes auf die Qualität des Milchentzuges in einem umfangreichen, Tierexperiment. 2. Euterentleerungsgrad und Melkgesch windigkeit, Milchwissenschaft 38, 276-279

McGrath D.M. \& O'Shea J. (1972) Effect of teatcup liner design on milking characteristics. Ir. J. Agric. Res. 11, 339-349

Mein G.A. \& Williams D.M. (1984) Liner massage and teat condition. Proc. $23^{\text {rd }}$ in Meeting Nat. Mastitis Council, Kansas City, USA (Nat. Mast. Council. ed.) Inc. Washington DC, USA, pp. 4-18

O'Shea J. (1987) Machine milking factors affecting mastitis - a literature review in Machine milking and Mastitis. Bull. I.D.F. $\mathrm{n}^{\circ} 215,1-34$, Ed. F.I.L., Brussels

O'Shea J. \& O'Callaghan E. (1980) Milking characteristics of 30 cluster types. In : Experiments on milking machine components at Moorepark 1976-1979 (O'Shea and O'Callaghan eds.) An Foras Taluntais, Dublin, pp. 170191

Schneider C. (1980) Influence des différentes machines à traire sur la consistance et la température du bout du trayon de la vache. Thèse Méd. Vét. Université de Berne

Thiel C.C. (1974) Mechanics of the action of the milking machine cluster. Biennal Reviews, Nat. Institute for Res. in Dairying, pp. 35-58

Williams D.M. \& Mein G.A. (1982) Review : physical and physiological factors affecting milk flow rate from the bovine teat during machine milking. Proc. Conf. on Dairy Production from Pasture, (K.L. Macmillan and V.K. Taufa eds) Hamilton, New Zealand, pp. 42-74 